\title{
Evaluation of the effect of oral administration of collagen peptides on an experimental rat osteoarthritis model
}

\author{
SATOKO ISAKA $^{1,2}$, AKIMASA SOMEYA ${ }^{3}$, SHINJI NAKAMURA $^{4}$, KIYOHITO NAITO ${ }^{1}$, MASAHIKO NOZAWA ${ }^{1,2}$, \\ NAOKI INOUE $^{5}$, FUMIHITO SUGIHARA ${ }^{5}$, ISAO NAGAOKA ${ }^{3}$ and KAZUO KANEKO ${ }^{1}$ \\ ${ }^{1}$ Department of Medicine for Motor Organ, Juntendo University Graduate School of Medicine, Tokyo 113-8421; \\ ${ }^{2}$ Department of Orthopaedic Surgery, Juntendo University, Nerima Hospital, Tokyo 117-8521; \\ ${ }^{3}$ Department of Host Defense and Biochemical Research; ${ }^{4}$ Laboratory of Morphology and Image Analysis, \\ Biomedical Research Center, Juntendo University Graduate School of Medicine, \\ Tokyo 113-8421; ${ }^{5}$ Nitta Gelatin Inc., Osaka 556-0022, Japan
}

Received December 27, 2015; Accepted January 26, 2017

DOI: $10.3892 /$ etm.2017.4310

\begin{abstract}
Collagen is an extracellular matrix protein present in the skin, tendon, cartilage and bone. Collagen peptides (CP) are produced by the hydrolysis of gelatin (heat-denatured collagen) by proteases and are utilized as a component of nutraceuticals. The current study investigated the effect of $\mathrm{CP}$ on the articular cartilage of OA by evaluating the serum levels of biomarkers (CTX-II for type II collagen degradation and CPII for type II collagen synthesis), histopathological changes (Mankin score, based on the toluidine blue staining of proteoglycans), and immunohistochemical staining of matrix metalloproteinase (MMP)-13 and type II collagen, using a rat experimental osteoarthritis (OA) model. Anterior cruciate ligament transection (ACLT) was performed on the right knee joint to surgically induce OA. Animals were divided into four groups: Control group (Control), sham-operated group (Sham), ACLT group without collagen peptide (ACLT group) and ACLT group with oral administration of CP (CP group). ACLT induced histological damages and significantly increased the Mankin score $(\mathrm{P}<0.05)$. However, $\mathrm{CP}$ administration markedly suppressed the Mankin score, although this difference was not significant. In addition, serum CTX-II levels were significantly decreased in CP group compared with those in the ACLT group $(\mathrm{P}<0.05)$. By contrast, serum CPII levels did not differ significantly among the four groups. Moreover, immunohistochemical staining of type II collagen and MMP-13 (an important type II collagen-degrading enzyme) indicated that the amount of type II collagen increased, whereas the
\end{abstract}

Correspondence to: Professor Isao Nagaoka, Department of Host Defense and Biochemical Research, Juntendo University Graduate School of Medicine, 2-1-1 Hongo, Bunkyo-ku, Tokyo 113-8421, Japan

E-mail: nagaokai@juntendo.ac.jp

Key words: collagen peptides, osteoarthritis, type II collagen, biomarkers, matrix metalloproteinase-13 number of MMP-13 positive chondrocytes decreased in the CP group compared with ACLT group. These observations suggest that $\mathrm{CP}$ has the potential to exert chondroprotective action on OA by inhibiting MMP-13 expression and type II collagen degeneration.

\section{Introduction}

Osteoarthritis (OA) is a chronic joint disease characterized by degeneration of articular cartilage, oseophyte formation and sclerosis of subchondral bone. These pathological changes cause joint stiffness, chronic pain and significant disability for patients with OA. Currently, a number of treatments are available for OA, from conservative treatments to surgical extremes, such as total knee arthroplasty (1). Conservative measures to treat OA involve pharmacological treatment with nonsteroidal anti-inflammatory drugs, intra-articular injection of hyaluronan and physical therapy (2). However, such treatments only reduce the pain experienced and do not treat the underlying pathology of the disease. Furthermore, they sometimes result in the development of adverse effects following long-term usage (3). Therefore, alternative approaches of managing OA need to be developed. Previous studies have investigated a number of nutraceuticals derived from functional foods that are often related to health-promotion or disease prevention, including glucosamine, which have a protective effect on cartilage $(4,5)$.

Collagen is an extracellular matrix protein localized in the skin, tendon, cartilage and bone (6). It has a unique triple helix configuration with a repeating amino acid sequence, (Gly-X-Y) $\mathrm{n}$, where $\mathrm{X}$ and $\mathrm{Y}$ are typically proline and hydroxyproline, respectively (7). Collagen peptides $(\mathrm{CP})$ are produced by the hydrolysis of gelatin (heat-denatured collagen) by proteases and are commonly utilized as a component of nutraceuticals (8). It has been demonstrated that, following the digestion of orally administered CP in the intestine, di- and tri-peptides, particularly Prolyl-hydroxyproline (Pro-Hyp), as well as amino acids, accumulate in human peripheral blood and these peptides remain in the blood for a relatively long time (3-4 h) (9-11). Pro-Hyp is considered to be a biologically active 
substance, as it induces hyaluronic acid synthesis in cultured synovial cells (12) and suppresses the hypertrophic differentiation of chondrocytes, which is involved in the pathogenesis of OA (13). In this context, it is interesting to note that oral administration of $\mathrm{CP}$ reduces the severity of symptoms experienced by patients with OA, reducing the Western Ontario McMaster University score and visual analogue scale, and improving the quality of life scores in patients with knee OA (14). Thus, CP is expected to be a component of functional foods that may be used to treat OA.

A number of OA models using aging animals and animals with spontaneous OA, such as STR/Ort mice, as well as animals with surgically, enzymatically or chemically induced OA (15), have been utilized to investigate the pathogenesis of OA and evaluate the potential of newly developed disease/structure-modifying drugs (16-19). The pathology and pathogenesis of spontaneous animal models of OA, including naturally occurring or transgenically-induced models, are considered to be similar to those in human OA (20). However, naturally occurring or transgenically-induced OA progresses slowly, meaning that testing protective agents or studying pathogenesis is time-consuming (20). In contrast, an induced anterior cruciate ligament transaction (ACLT) model of OA has been used to analyze the histopathological and biochemical changes occurring during OA progression (20). This is due to the fact that ACLT results in joint instability and induces cartilage degeneration, subchondral bone sclerosis and osteophyte formation, thus mimicking the pathological changes occurring in human OA (21). Therefore, the current study utilized the ACLT model to evaluate the effect of collagen peptides on OA.

The aim of the present study was to investigate the effect of $\mathrm{CP}$ on articular cartilage in OA by measuring levels of serum biomarkers for type II collagen, including CTX-II (type II collagen degradation) and CPII (type II collagen synthesis), as well as the histopathological changes occurring, in experimental ACLT model rats. Additionally, the effect of CP on type II collagen degradation and MMP-13 expression was investigated using immunohistochemical staining.

\section{Materials and methods}

Animal model and collagen peptide administration. A total of 28 male Sprague-Dawley rats (age, 10 weeks; weight, $320.6 \pm 1.9 \mathrm{~g}$ ) purchased from Charles River Breeding Laboratories (Yokohama, Japan) were used in the present study.

OA was surgically induced in the right knee joint. Each rat was anesthetized with $2 \%$ isoflurane (Pfizer Inc., Tokyo, Japan) in air with a delivery rate of $0.21 / \mathrm{min}$ and ketamine hydrochloride $(58 \mathrm{mg} / \mathrm{kg}$; Daiichi Sankyo Co., Ltd., Tokyo, Japan). Following anesthetization, the right knee joint was exposed through a medial parapatellar approach after the knee was shaved and disinfected with $10 \%$ povidone-iodine solution (Yoshida Pharmaceutical Co., Ltd., Tokyo, Japan). The patella was dislocated laterally and the knee placed in full flexion and ACLT was performed with micro scissors, as previously described (21). Following surgery, the joint surface was rinsed with sterile saline and both capsule and skin were sutured using Vicryl 4-0 absorbable suture (Ethicon US, LLC., Somerville, NJ, USA) and monofilament 4-0 Nylon threads (Monosof ${ }^{\mathrm{TM}}$; Medtronic, Minneapolis, MN, USA), respectively. In sham-operated animals, the right knee joint was exposed and incisions were closed following subluxation of the patella and rinsing of the joint surface with saline.

CP was supplied by Nitta Gelatin, Inc. (Osaka, Japan). Standard laboratory diet (CRF-1) containing 12\% CP was prepared by Oriental Yeast Co., Ltd. (Tokyo, Japan). Standard laboratory diet with or without $\mathrm{CP}$ was available to rats ad libitum for 8 weeks following the surgery. Food intake and body weights were measured once a week. Food intake ( $\mathrm{g} / \mathrm{rat} /$ week) was calculated by dividing the weight of diet consumed in a week by the number of rats in a cage.

Rats were randomly divided into the four groups (all $\mathrm{n}=7)$ : i) Control group (Control), ii) sham-operated group (Sham group), iii) ACLT group without CP (ACLT group) and iv) ACLT group with CP (CP group). All groups (Control, Sham, ACLT and CP) were allowed to move freely in plastic cages. Two or three rats were housed per cage under specific pathogen-free conditions (controlled temperature of $24 \pm 3^{\circ} \mathrm{C}$ and humidity of $55 \pm 15 \%$ ) with a 12 -h light/dark cycle and had ad libitum access to tap water. Animals were sacrificed 56 days following surgery by an intramuscular injection of $60 \mathrm{mg} / \mathrm{kg}$ pentobarbital sodium (Somnopentyl; Kyoritsu Seiyaku Corp., Tokyo, Japan). All procedures performed were approved by the Institutional Animal Care and Use Committee of Juntendo University School of Medicine (Tokyo, Japan; 260160). All animal experiments were carried out in compliance with the guidelines for animal experimentation of Juntendo University School of Medicine and with relevant federal laws and regulations.

Tissue preparation and histopathological evaluation. Histopathological evaluation was conducted on the sagittal sections of cartilage in the weight-bearing area of the medial femoral condyle and the medial tibia plateau. Following disarticulation, knee joint samples were cleaned and fixed in $10 \%$ formalin at room temperature for $24 \mathrm{~h}$, decalcificated using Gooding and Stewart's fluid $(300 \mathrm{ml}$ each of $10 \%$ formalin and $10 \%$ formic acid solution; Wako Pure Chemical Industries, Ltd., Osaka, Japan) and embedded in paraffin. Sections 5- $\mu \mathrm{m}$ thick were stained with $0.05 \%$ toluidine blue $(\mathrm{pH} \mathrm{4.1)}$ and the severity of the OA lesion was graded on a scale of $0-13$, using the modified Mankin scoring system $(14,17)$. The Mankin scoring system generated a combined score of structure ( $0-6$ points, with 0 points representing normal and 6 points representing complete disorganization), cellular abnormalities (0-3 points, with 0 points representing normal and 3 points representing hypocellularity) and matrix staining ( $0-4$ points, with 0 points representing normal staining and 4 points indicating that no dye was noted). Histopathological evaluation was performed by two independent blinded observers.

Enzyme linked immunoassay (ELISA) of serum biomarkers. Blood samples were obtained from the heart by cardiac puncture prior to and 7, 14, 28 and 56 days following surgery, and sera were obtained by centrifugation $(1,000 \times \mathrm{g})$ for $10 \mathrm{~min}$ at $4^{\circ} \mathrm{C}$ and stored in aliquots at $-80^{\circ} \mathrm{C}$. Serum levels of the type II collagen degradation marker CTX-II (22) were measured using a serum Pre-Clinical CartiLaps ELISA kit (AC-08F1; Nordic Bioscience Diagnostic A/S, Herlev, Denmark). Serum 
levels of the type II collagen synthesis biomarker CPII (22) were measured using a Procollagen type II C-propeptide ELISA kit (60-1003-001; IBEX Technologies Inc., Montreal, Canada). CPII is cleaved from type II procollagen during the processing of newly synthesized procollagen and thus can be used as a type II collagen synthesis marker.

Immunohistochemical staining of type II collagen and MMP-13. Rabbit anti-collagen type II (ab34712) and anti-matrix metalloproteinase (MMP)-13 (ab75606) antibodies were purchased from Abcam (Cambridge, MA, USA). Deparaffinized sections were digested with proteinase K (Dako; Agilent Technologies, Inc., Santa Clara, CA, USA) to obtain optimal antigen exposure for $30 \mathrm{~min}$ at room temperature. Following two washes with phosphate-buffered saline (PBS; pH 7.2), 5\% normal goat serum (Vector Laboratories, Inc., Burlingame, CA, USA) was used to block non-specific binding sites for $20 \mathrm{~min}$ at room temperature. Sections were incubated with anti-collagen type II or anti-MMP-13 antibody [1:100 dilution in 2\% bovine serum albumin (Sigma-Aldrich; Merck KGaA, Darmstadt, Germany) in PBS] at $4^{\circ} \mathrm{C}$ overnight. Subsequently, slides were washed with PBS and incubated with biotinylated goat anti-rabbit immunoglobulin G (1:300; E432; Dako; Agilent Technologies, Inc.) for $30 \mathrm{~min}$ at room temperature, followed by incubation with peroxidase-conjected avidin (Dako; Agilent Technologies, Inc.) for $30 \mathrm{~min}$ at room temperature. Peroxidase activity was detected using 3'3-diaminobenzidine tetrahydrochloride (Dojindo Molecular Technologies, Inc., Kumamoto, Japan). Immunologically stained areas were analyzed using the Tissue Studio (Definiens AG, Munich, Germany) computer imaging system. Type II collagen was immunohistochemically stained primarily in the superficial areas of cartilage, and its staining was quantitatively evaluated and expressed as arbitrary units $/ \mathrm{mm}^{2}$ by dividing the intensities of the staining areas by the gross area of cartilage in each section. By contrast, immunohistochemical staining of MMP-13 primarily occurred in the chondrocytes and the number of MMP-13 positive chondrocytes was counted and expressed as cells $/ \mathrm{mm}^{2}$ by dividing the number of MMP-13 positive chondrocytes by the gross area of cartilage in each section.

Statistical analyses. Data are expressed as mean \pm standard deviation. Statistical analysis was performed with one-way analysis of variance using a StatView 5.0 program (SAS Institute Inc., Cary, NC, USA). The values of seven animals in each group were compared between the Control, Sham, ACLT and $\mathrm{CP}$ groups. $\mathrm{P}<0.05$ was considered to represent a statistically significant difference.

\section{Results}

Evaluation of body weight and food intake. During the experimental period, there was a similar increase in the body weight of animals over time in all four groups (Control, Sham, ACLT and $\mathrm{CP}$ ). Therefore, it is evident that administration of $\mathrm{CP}$ did not affect the body weights of the animals (Fig. 1A). During the first week (days 1-7) after the operation, food intake was reduced in Sham, ACLT, and CP groups compared with Control group, possibly due to surgical stress; however, this reduction was not statistically significant (Fig. 1B). After 1 week, food
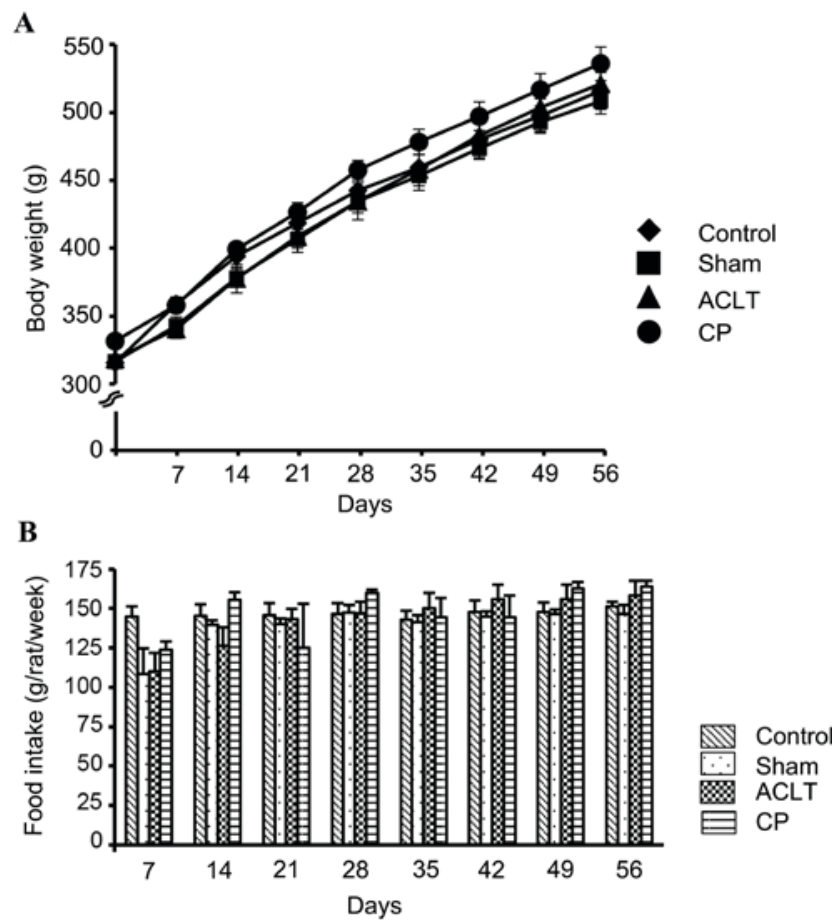

Figure 1. Body weight change and food intake in a rat osteoarthritis model. (A) Body weight changes during the experimental period in Control, Sham, ACLT, and CP groups. (B) Food intake (g/rat/week) in the Control, Sham, ACLT and CP groups. Data represent the mean \pm standard deviation of seven animals in each group. ACLT, anterior cruciate ligament transaction model; $\mathrm{CP}$, collagen peptides.

intake gradually increased with age; the average food intake of the four groups was $121.82 \mathrm{~g} / \mathrm{rat} /$ week in the first week (days 1-7), and $155.07 \mathrm{~g} / \mathrm{rat} /$ week in the last week (days 50-56). During the experimental period (days 1-56), food intake did not differ significantly among the four groups (Control, Sham, ACLT and CP), and administration of CP did not affect food intake. Based on the body weight and food intake, it was determined that animals in the $\mathrm{CP}$ group received 5.0-6.7 $\mathrm{g} / \mathrm{kg} /$ day $\mathrm{CP}$ during the experimental period.

Evaluation of the effect of CP on the gross morphology and the histopathological changes in articular cartilage. No macroscopic degenerative changes, such as erosion and osteophyte formation, were detected on the articular surfaces of femoral condyles and tibia plateau in the animals in any of the four groups (data not shown). However, ACLT clearly induced histopathological changes (Fig. 2), including surface irregularity of the medial femoral condyle (Fig. 2E) and tibia plateau (Fig. 2F), surface depletion and reduced toluidine blue staining in the cartilage (ACLT vs. Control and Sham; Fig. 2A-F). Notably, administration of CP improved the surface irregularity and toluidine blue staining in the cartilage (Fig. 2G and $\mathrm{H}$ ). These changes were further evaluated using the Mankin score. As expected, the total Mankin score was significantly higher in the ACLT group compared with the Control group in the femoral condyles and tibia plateau $(\mathrm{P}<0.05$; Fig. 3). Furthermore, the Mankin score was substantially reduced in the CP group compared with the ACLT group; however, this reduction was not statistically significant 

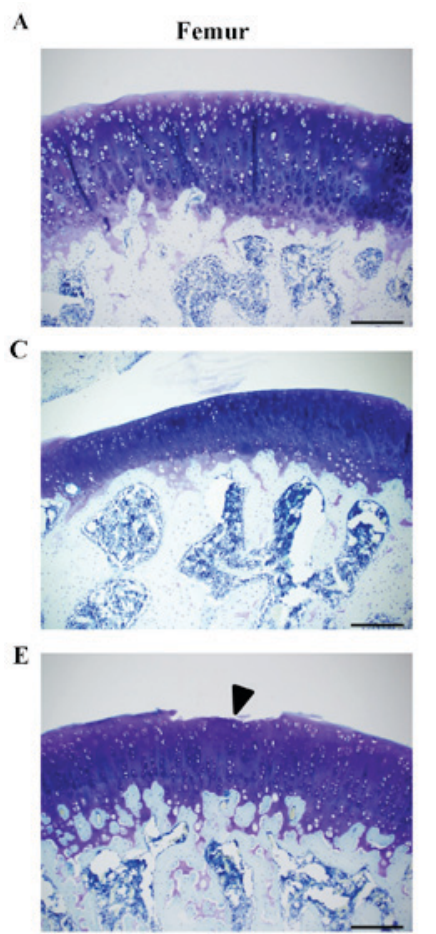

G

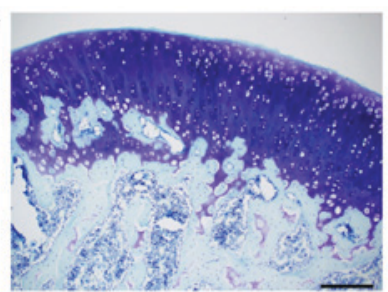

B

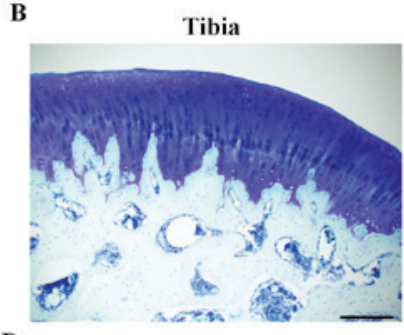

D
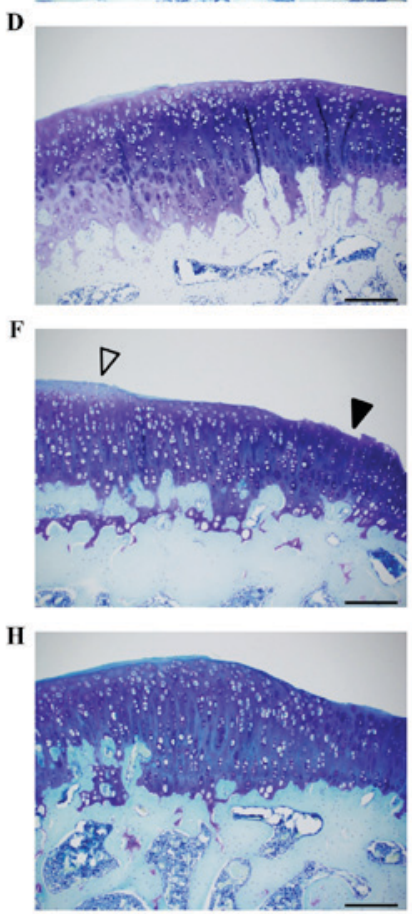

Figure 2. (A-H) Histopathological evaluation of the articular cartilage in a rat osteoarthritis model. Knee joints were dissected 56 days following the ACLT operation and the sagittal sections of cartilage in the weight-bearing area of the medial femoral condyle and the medial tibia plateau were stained with toluidine blue. (A) Control, (C) Sham, (E) ACLT and (G) CP in the femoral condyle and (B) Control, (D) Sham, (F) ACLT and (H) CP in the tibia plateau. ACLT induced the irregularity and surface depletion (closed arrow head) in the (E) medial femoral condyle and the (F) medial tibia plateau, and the reduction of toluidine blue-staining (open arrowhead) in the (F) medial tibia plateau. Scale bar $=200 \mu \mathrm{m}$. ACLT, anterior cruciate ligament transaction model; $\mathrm{CP}$, collagen peptides.

$(\mathrm{P}=0.1)$. Thus, administration of $\mathrm{CP}$ improved the histopathological change of cartilage in an OA model.

Evaluation of biomarkers. Subsequently, the effect of CP administration on articular cartilage was investigated using biomarkers for type II collagen degradation and synthesis. In a preliminary experiment, it was confirmed that the administration of CP did not influence serum levels of the biomarkers CTX-II and CPII (data not shown).

Pre-operational levels of CTX-II and CPII did not differ significantly among the four groups (Control, Sham, ACLT and CP; Fig. 4). Over time, CTX-II levels decreased in all groups. However, the CTX-II level was lower in the CP group compared with the ACLT group 7-56 days following operation, and CTX-II levels were significantly reduced in the $\mathrm{CP}$ group compared with the ACLT group 56 days after the operation $(\mathrm{P}<0.05$; Fig. $4 \mathrm{~A})$. Levels of CTX-II at 56 days were $21.13 \pm 22.89 \mathrm{ng} / \mathrm{ml}$ in the ACLT group and $0.04 \pm 0.11 \mathrm{ng} / \mathrm{ml}$ in the $\mathrm{CP}$ group. This indicates that

A

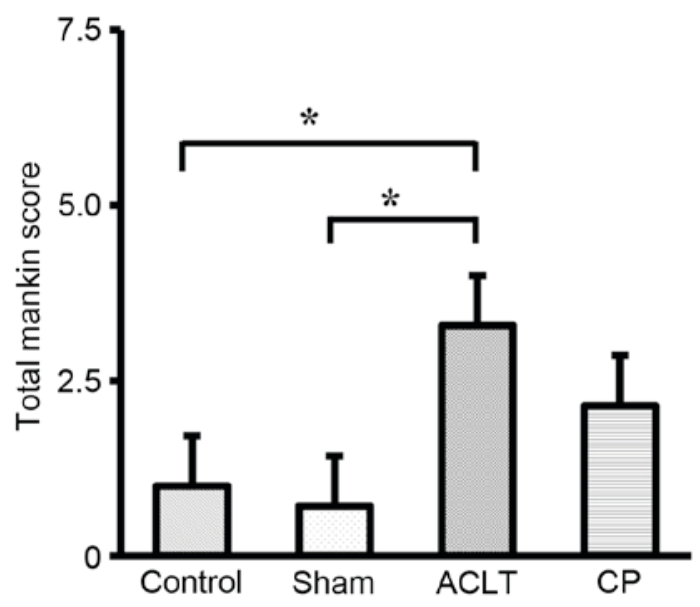

B

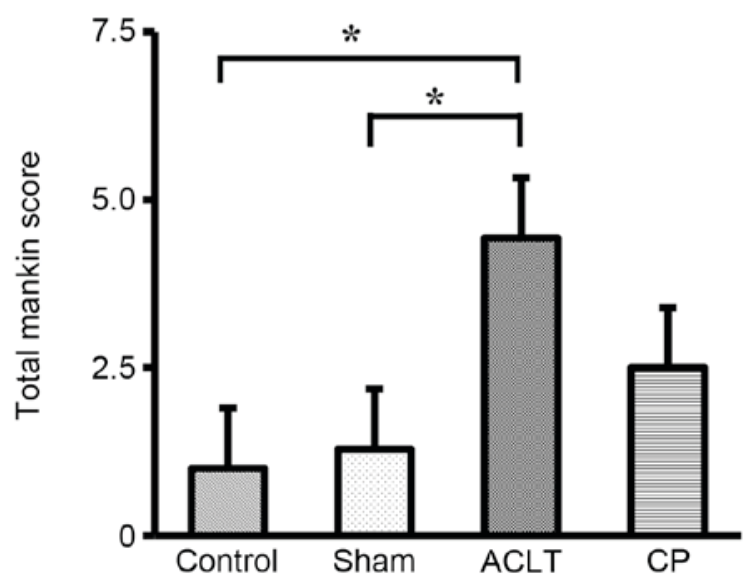

Figure 3. Grading of the severity of OA lesion with the modified Mankin scoring system in a rat OA model. The severity of OA lesion in (A) femoral condyle and (B) tibia plateau was graded on a scale of 0-13 using the modified Mankin scoring system. Data represents the mean \pm standard deviation of seven animals in each group (Control, Sham, ACLT and CP). Values were compared between the ACLT and Control groups, or the ACLT and Sham groups. ${ }^{*} \mathrm{P}<0.05$. OA, osteoarthritis; ACLT, anterior cruciate ligament transaction model; $\mathrm{CP}$, collagen peptides.

the administration of CP suppresses the ACLT-induced degradation of type II collagen. By contrast, there was no significant change in CPII levels among the four groups throughout the experimental period of 56 days (Fig. 4B).

Type II collagen staining in articular cartilage. The effect of $\mathrm{CP}$ administration on the biomarkers suggests that $\mathrm{CP}$ suppresses the ACLT-induced degradation of type II collagen in the articular cartilage. To test this hypothesis, type II collagen was immunohistochemically stained and the effect of CP administration was evaluated (Fig. 5). Type II collagen was less stained in the femoral condyle of the ACLT group compared with the Control and Sham groups (Fig. 5E). Interestingly, type II collagen staining was enhanced in the femoral condyle of CP group (Fig. 5G) compared with the ACLT group (Fig. 5E). By contrast, the staining of type II collagen in the tibia plateau was similar among all four groups (Fig. 5B, D, F and H). 
A

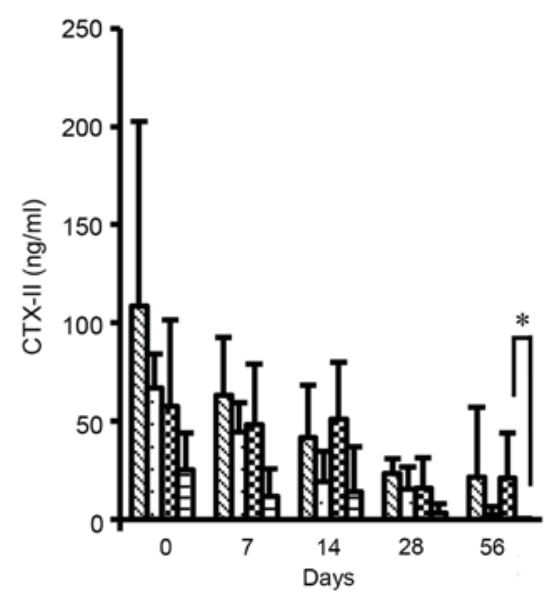

B

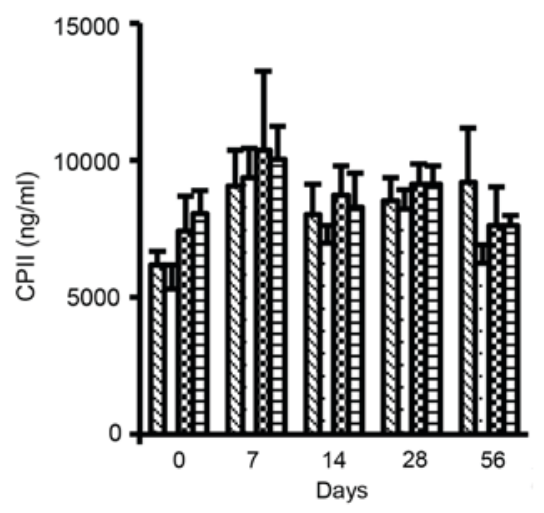

A
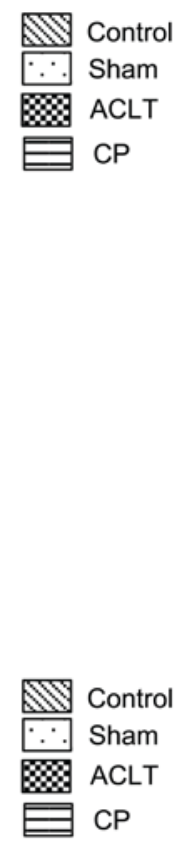

E

Figure 4. Effects of CP administration on the biomarkers for type II collagen in a rat osteoarthritis model. Sera were prepared prior to (0 day) and 7, 14, 28 and 56 days following surgery and used for (A) CTX-II and (B) CPII assays. Pre-operational levels of CTX-II and CPII were similar among the four groups (Control, Sham, ACLT and CP). However, levels of CTX-II 56 days following surgery were significantly lower in the $\mathrm{CP}$ group compared with the ACLT group. Data represent the mean \pm standard deviations of seven animals in each group. Values were compared between the ACLT and CP groups. ${ }^{*} \mathrm{P}<0.05$. OA, osteoarthritis; ACLT, anterior cruciate ligament transaction model; CP, collagen peptides; CTX-II, type II collagen degradation marker; CPII, type II collagen synthesis biomarker.

Consistent with these observations, quantification of immunohistochemical staining of type II collagen indicated that type II collagen staining in the femoral condyle was reduced in the ACLT group compared with the control group, but recovered in the CP group, although none of these changes were significant (Fig. 6A). Type II collagen staining was similar among all four groups in the tibia plateau (Fig. 6B).

MMP-13 staining in articular cartilage. MMP-13 is one of the major enzymes involved in the type II collagen degradation and its expression is upregulated in OA (23). Thus, MMP-13 was immunohistochemically stained and evaluated. Fig. 7 shows that the number of MMP-13 positive chondrocytes in the femoral condyle was clearly increased not only in the ACLT group, but also the Sham group compared with the Control group (Fig. 7A, C and E). Importantly, the number of MMP-13 positive chondrocytes was decreased in the $\mathrm{CP}$ group (Fig. $7 \mathrm{G}$ ) compared with the Sham and ACLT groups. The number of
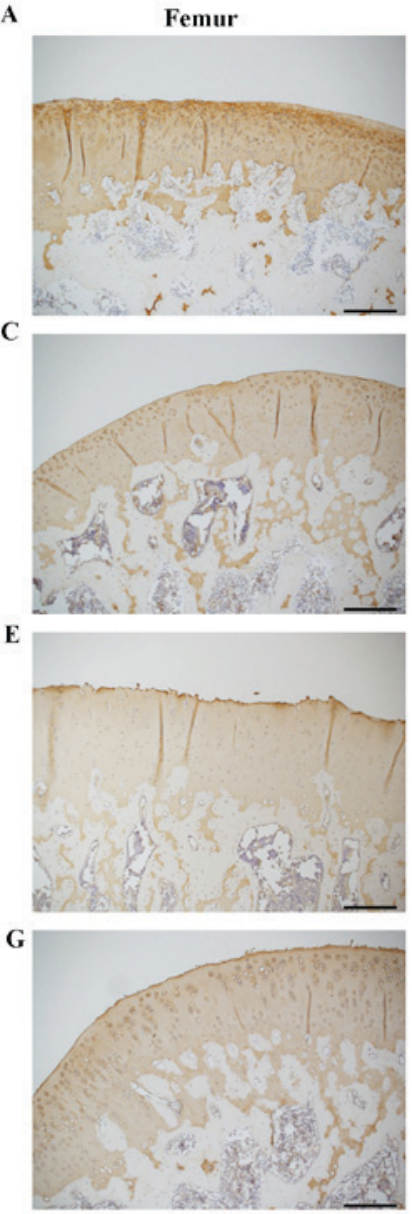

B

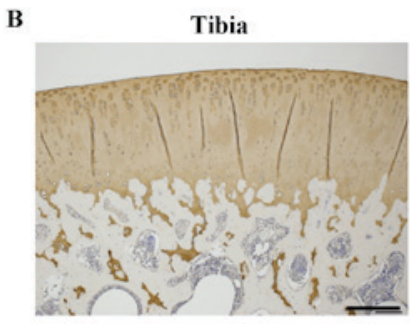

D
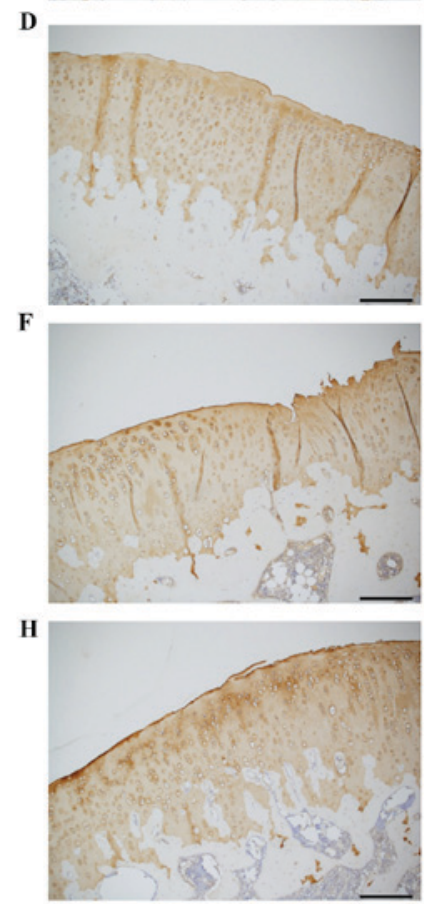

Figure 5. (A-H) Immunohistochemical staining of type II collagen in the knee joints of a rat osteoarthritis model. Type II collagen was immunohistochemically stained in the sagittal sections of cartilage in the weight-bearing area of the femoral condyle (A, Control; C, Sham; E, ACLT; G, CP) and the medial tibia plateau (B, Control; D, Sham; F, ACLT; H, CP). Scale bar $=200 \mu \mathrm{m}$. Photos are representative of seven animals in each group and present the enlarged area of each section. ACLT, anterior cruciate ligament transaction model; CP, collagen peptides; CTX-II, type II collagen degradation marker; CPII, type II collagen synthesis biomarker.

MMP-13 positive chondrocytes was similar among the Control, Sham, and ACLT groups in the tibia plateau (Fig. 7B, D, and F). Interestingly, however, the number of MMP-13 positive chondrocytes was slightly decreased in the $\mathrm{CP}$ group (Fig. $7 \mathrm{H}$ ).

Consistent with these observations, quantification of MMP-13 positive chondrocytes in the femoral condyle indicated that the number of MMP-13 positive chondrocytes was significantly increased in the Sham and ACLT groups compared with the Control group (both $\mathrm{P}<0.05$ ) and significantly decreased in the CP group compared with the Sham and ACLT groups $(\mathrm{P}<0.05 ;$ Fig. 8A). However, there were no significant differences in the number of MMP-13 positive chondrocytes among Sham, Control, ACLT and CP groups in the tibia plateau, although there was a slight decrease in the number of MMP-13 positive chondrocytes in the $\mathrm{CP}$ group (Fig. 8B).

\section{Discussion}

In recent years, it has been demonstrated that oral administration of $\mathrm{CP}$ protects against OA. To determine the mechanism 
A

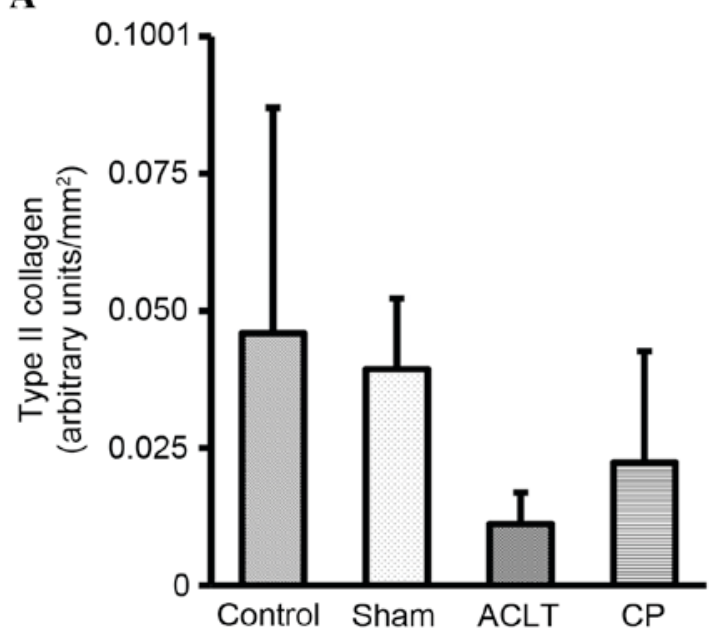

B

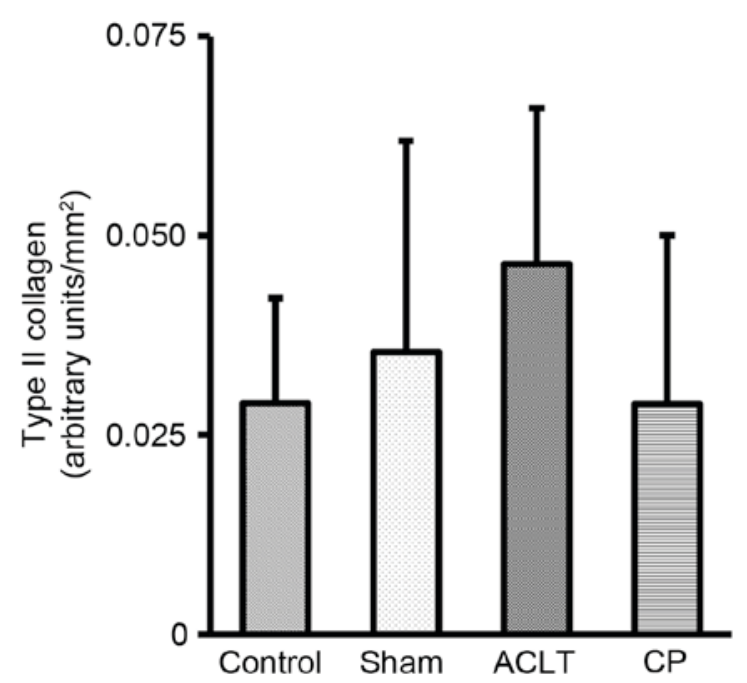

Figure 6. Quantification of the immunohistochemical staining of type II collagen in the knee joints of a rat osteoarthritis model. The intensities of the type II collagen staining were analyzed, and expressed as arbitrary units $/ \mathrm{mm}^{2}$ in the (A) femoral condyle and the (B) tibia plateau. There were no significant differences in the staining intensities of type II collagen among all groups. Data represent the mean \pm standard deviation of seven animals in each group. ACLT, anterior cruciate ligament transaction model; $\mathrm{CP}$, collagen peptides.

of $\mathrm{CP}$ action on the articular cartilage, the current study evaluated the serum levels of two biomarkers, CTX-II and CPII, histopathological changes, and immunohistochemical staining of type II collagen and MMP-13 in an experimental OA rat model. The results indicated that ACLT significantly induced histological damage and increased the Mankin score. However, CP administration substantially suppressed the Mankin score. Serum CTX-II levels were significantly decreased in the CP group compared with the ACLT group, however serum CPII levels did not differ significantly among the four groups. Furthermore, immunohistochemical staining of type II collagen and MMP-13 indicated that the amount of type II collagen was increased, whereas the number of MMP-13 positive chondrocytes was decreased in the CP group compared with the ACLT group.
$\mathbf{A}$

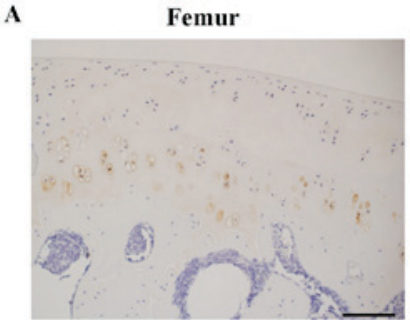

C

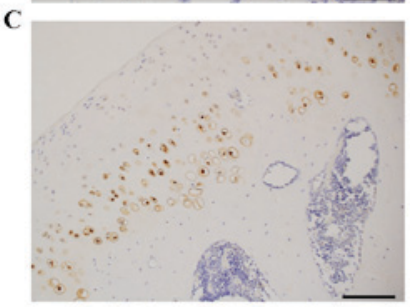

$\mathbf{E}$

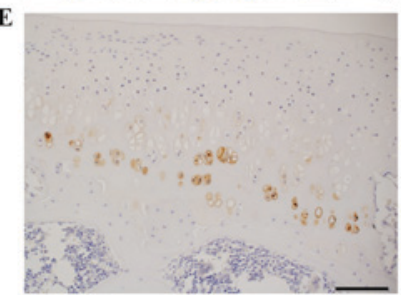

G

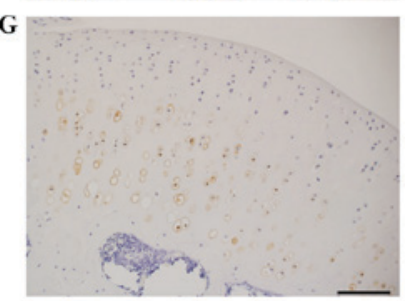

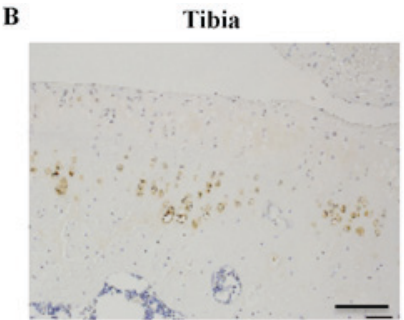

D
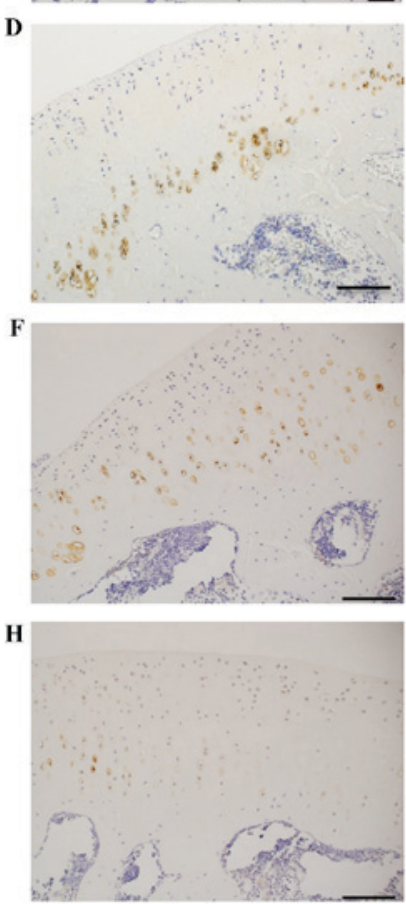

Figure 7. (A-H) Immunohistochemical staining of MMP-13 positive chondrocytes in the knee joints of a rat osteoarthritis model. MMP-13 was immunohistochemically stained in the sagittal sections of cartilage in the weight-bearing area of the femoral condyle (A, Control; C, Sham; E, ACLT; $\mathrm{G}, \mathrm{CP}$ ) and the medial tibia plateau (B, Control; D, Sham; F, ACLT and H, $\mathrm{CP})$. MMP-13 positive chondrocytes were detected in these sections. Scale bar $=200 \mu \mathrm{m}$. Photos are representative of seven animals in each group and present the enlarged area of each section. MMP-13, matrix metalloproteinase 13; ACLT, anterior cruciate ligament transaction model; CP, collagen peptides.

Previous clinical studies have suggested that $\mathrm{CP}$ (4.5-10 g/day) has a protective effect on the joints $(8,14,24,25)$. For example, it has been demonstrated that administration of $10 \mathrm{~g} \mathrm{CP} /$ day for 2 months relieves knee and hip joint pain in patients with $\mathrm{OA}(8)$ and that administration of $10 \mathrm{~g} \mathrm{CP} /$ day for 24 weeks reduces activity-related joint pain in athletes (24). Based on these findings, $0.166 \mathrm{~g} / \mathrm{kg}$ body weight/day $\mathrm{CP}$ (10 g/60 kg/day) is estimated to be effective at treating joint pain in humans. In rats however, it has been reported that the administration of $1.66 \mathrm{~g} / \mathrm{kg} /$ day CP increases the bone mineral density of the femoral bone and lumbar spine (26). Therefore, in the present study, to more precisely determine the effect of $\mathrm{CP}$ on the cartilage, a dose of 5.0-6.7 g/ kg/day CP, 30-40-fold higher than the effective dose in humans $(0.166 \mathrm{~g} / \mathrm{kg} /$ day $)$ was administered to ACLT rats.

Currently, researchers are trying to evaluate OA using the constituents of cartilage, including aggrecan, chondroitin sulfate and collagen, as biomarkers (22). Type II collagen is a major component of articular cartilage, representing 90-95\% of total collagen content and forming the fibrils that give 
A

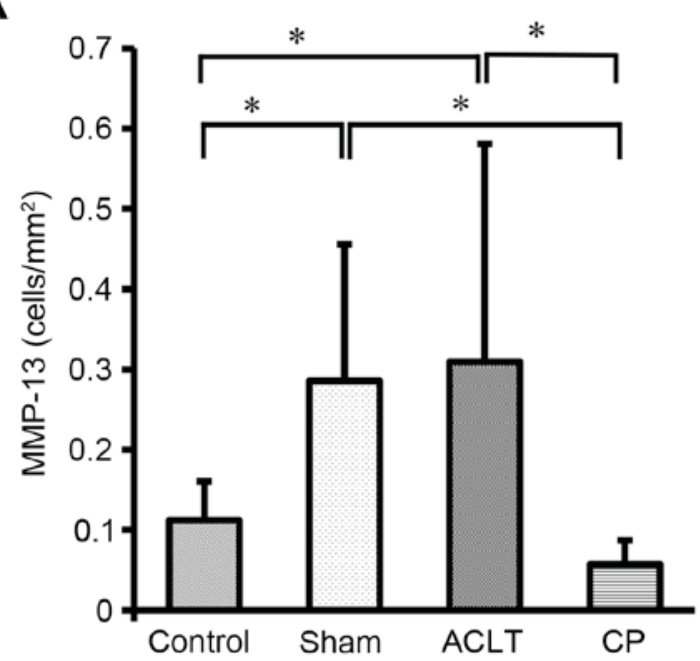

B

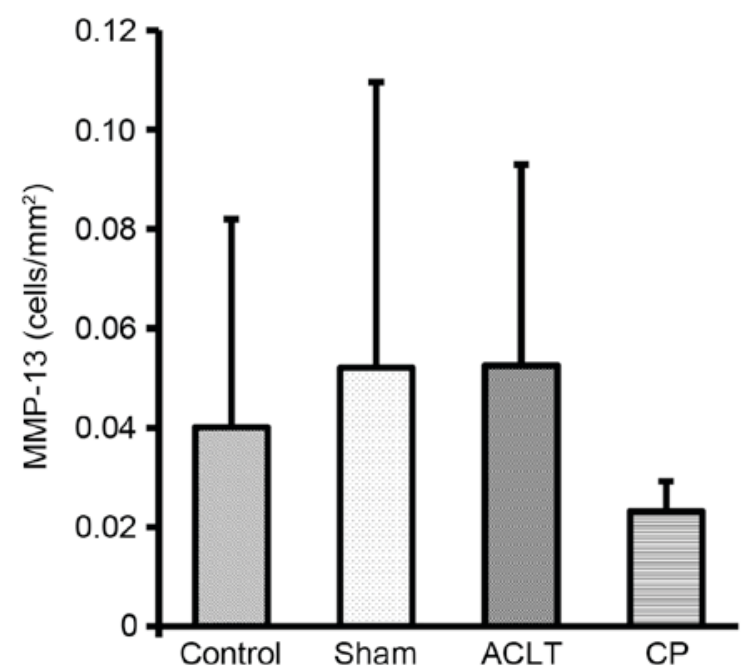

Figure 8. Quantification of the MMP-13 positive chondrocytes in the knee joints of a rat osteoarthritis model. MMP-13 positive chondrocytes were counted and expressed as cells $/ \mathrm{mm}^{2}$ in the (A) femoral condyle and (B) the tibia plateau. Data represent the mean \pm standard deviation of seven animals in each group. Values were compared among the four groups. ${ }^{*} \mathrm{P}<0.05$. MMP-13, matrix metalloproteinase 13; ACLT, anterior cruciate ligament transaction model; $\mathrm{CP}$, collagen peptides.

cartilage its tensile strength (23). Among the biomarkers that have been investigated (22), the components that make up type II collagen are recognized as the most important biomarkers for OA (23). This is due to the fact that type II collagen is specifically expressed in cartilage and OA essentially involves catabolism and anabolism of articular type II collagen. However, there have been no studies evaluating the effect of $\mathrm{CP}$ on OA using biomarkers. Thus, to the best of our knowledge, this is the first study to investigate the effects of $\mathrm{CP}$ on OA by analyzing cartilage metabolism using biomarkers for type II collagen.

The results of the present study revealed that the level of CTX-II was reduced in the CP group compared with the ACLT group during the 7-56 days after operation, suggesting that CP suppresses the degradation of type II collagen during OA progression in this model. Indeed, immunohistochemical staining for type II collagen indicated that type II collagen levels are maintained in the CP group compared with the ACLT group in the femoral condyle. Furthermore, immunohistochemical staining for MMP-13, a major type II collagen-degradation enzyme involved in the pathogenesis of OA, (23) indicated that the number of MMP-13 positive chondrocytes was decreased in the $\mathrm{CP}$ group compared with the ACLT group. Taken together, these observations suggest that $\mathrm{CP}$ potentially suppresses the expression of MMP-13 and the degradation of type II collagen in the articular cartilage of ACLT rats.

Following oral administration of CP, Pro-Hyp accumulates in human peripheral blood and remains in the blood for a relatively long time (3-4 h) (9-12,14,27). Importantly, the administration of Pro-Hyp suppresses the morphological change and cartilage destruction in the knee joints of animal OA models $(10,11)$. Furthermore, results from an in vitro study demonstrated that Pro-Hyp suppresses the hypertrophic differentiation of chondrocytes, which is observed in the pathogenesis of OA and stimulates glycosaminoglycan synthesis by chondrocytes (13). Pro-Hyp also stimulates the production of hyaluronic acid by cultured synovial cells $(12,28)$. Notably, hyaluronic acid exhibits anti-inflammatory action (29). Based on these findings, it is tempting to speculate that Pro-Hyp, which is derived from orally administered CP, enhances the production of hyaluronic acid and glycosaminoglycans by synovial cells and chondrocytes, thereby exhibiting anti-inflammatory and chondroprotective actions (as evidenced by the suppression of MMP-13 expression, type II collagen degradation and morphological changes) in vivo in our model.

In conclusion, the results of the present study suggest that $\mathrm{CP}$ has the potential to exert chondroprotective action on OA by inhibiting MMP-13 expression and type II collagen degeneration. Further studies investigating the components of $\mathrm{CP}$, such as Pro-Hyp, are required to elucidate the detailed mechanism behind the beneficial effect of $\mathrm{CP}$ on joint health.

\section{Acknowledgments}

The authors wish to thank Hiroshi Kawai (Division of Experimental Surgery and Biomedical Resources, Center for Biomedical Research Resources, Juntendo University Graduate School of Medicine) for his valuable advice on animal experiments, as well as Yuko Kojima, Atsushi Furuhata, Yasuko Toi and Katsumi Miyahara (Laboratory of Morphology and Image Analysis, Biomedical Research Center, Juntendo University Graduate School of Medicine) for their assistance of immunohistochemical staining and image analysis in the present study. Please note that two of the authors of the current study, Naoki Inoue and Fumihito Sugihara, are also employees at Nitta Gelatin, Inc., which is where the collagen peptides were purchased from. However, they had no competing financial interests in this research project. This study was supported in part by a grant from the Strategic Research Foundation Grant-aided Project for Private Universities from Ministry of Education, Culture, Sport, Science and Technology, Japan, 2014-2018 (grant no. S1411007). 


\section{References}

1. Tan D, Teh DJ, Bin Abd Razak HR and Tan AH: Improvement in health-related quality of life after unilateral total knee arthroplasty in patients with bilateral knee osteoarthritis. J Orthop Surg (Hong Kong) 24: 294-297, 2016.

2. Puhl W, Bernau A, Greiling H, Köpcke W, Pförringer W, Steck KJ, Zacher J and Scharf HP: Intra-articular sodium hyaluronate in osteoarthritis of the knee: A multicenter, double-blind study. Osteoarthritis Cartilage 1: 233-241, 1993.

3. Rashad S, Revell P, Hemingway A, Low F, Rainford K and Walker F: Effect of non-steroidal anti-inflammatory drugs on the course of osteoarthritis. Lancet 2: 519-522, 1989.

4. Nagaoka I: Recent aspects of the chondroprotective and anti-inflammatory actions of glucosamine, a functional food. Juntendo Med J 60: 580-587, 2014.

5. Clayton JJ: Nutraceuticals in the management of osteoarthritis. Orthopedics 30: 624-631, 2007.

6. Kawaguchi T, Nanbu PN and Kurokawa M: Distribution of prolylhydroxyproline and its metabolites after oral administration in rats. Biol Pharm Bull 35: 422-427, 2012.

7. Bos KJ, Rucklidge GJ, Dunbar B and Robins SP: Primary structure of the helical domain of porcine collagen X. Matrix Biol 18 149-153, 1999.

8. Moskowitz RW: Role of collagen hydrolysate in bone and joint disease. Semin Arthritis Rheum 30: 87-99, 2000.

9. Iwai K, Hasegawa T, Taguchi Y, Morimatsu F, Sato K, Nakamura Y, Higashi A, Kido Y, Nakabo Y and Ohtsuki K: Identification of food-derived collagen peptides in human blood after oral ingestion of gelatin hydrolysates. J Agric Food Chem 53: 6531-6536, 2005.

10. Ohara H, Matsumoto H, Ito K, Iwai K and Sato K: Comparison of quantity and structures of hydroxyproline-containing peptides in human blood after oral ingestion of gelatin hydrolysates from different sources. J Agric Food Chem 55: 1532-1535, 2007.

11. Sugihara F, Inoue N, Kuwamori $M$ and Taniguchi $M$ : Quantification of hydroxyprolyl-glycine (Hyp-Gly) in human blood after ingestion of collagen hydrolysate. J Biosci Bioeng 113 202-203, 2012 .

12. Ohara H, Iida H, Ito K, Takeuchi Y and Nomura Y: Effects of Pro-Hyp, a collagen hydrolysate-derived peptide, on hyaluronic acid synthesis using in vitro cultured synovium cells and oral ingestion of collagen hydrolysates in a guinea pig model of osteoarthritis. Biosci Biotechnol Biochem 74: 2096-2099, 2010.

13. Nakatani S, Mano H, Sampei C, Shimizu J and Wada M: Chondroprotective effect of the bioactive peptide prolyl-hydroxyproline in mouse articular cartilage in vitro and in vivo. Osteoarthritis Cartilage 17: 1620-1627, 2009

14. Kumar S, Sugihara F, Suzuki K, Inoue $\mathrm{N}$ and Venkateswarathirukumara S: A double-blind,placebo-controlled, randomised, clinical study on the effectiveness of collagen peptide on osteoarthritis. J Sci Food Agric 95: 702-707, 2015.

15. Bendele AM: Animal models of osteoarthritis. J Musculoskelet Neuronal Interact 1: 363-376, 2001.

16. Oegema TR Jr, Deloria LB, Sandy JD and Hart DA: Effect of oral glucosamine on cartilage and meniscus in normal and chymopapain-injected knees of young rabbits. Arthritis Rheum 46: 2495-2503, 2002.
17. Hoegh-Andersen P, Tankó LB, Andersen TL, Lundberg CV, Mo JA, Heegaard AM, Delaissé JM and Christgau S: Ovariectomized rats as a model of postmenopausal osteoarthritis: Validation and application. Arthritis Res Ther 6: R169-R180, 2004.

18. Jean YH, Wen ZH, Chang YC, Lee HS, Hsieh SP, Wu CT, Yeh CC and Wong CS: Hyaluronic acid attenuates osteoarthritis development in the anterior cruciate ligament-transected knee: Association with excitatory amino acid release in the joint dialysate. J Orthop Res 24: 1052-1061, 2006.

19. Tang T, Muneta T, Ju YJ, Nimura A, Miyazaki K, Masuda H, Mochizuki T and Sekiya I: Serum keratan sulfate transiently increases in the early stage of osteoarthritis during strenuous running of rats: Protective effect of intraarticular hyaluronan injection. Arthritis Res Ther 10: R13, 2008

20. Ameye LG and Young MF: Animal models of osteoarthritis: Lessons learned while seeking the 'Holy Grail'. Curr Opin Rheumatol 18: 537-547, 2006.

21. Hayami T, Pickarski M, Zhuo Y, Wesolowski GA, Rodan GA and Duong LT: Characterization of articular cartilage and subchondral bone changes in the rat anterior cruciate ligament transection and meniscectomized models of osteoarthritis. Bone 38: 234-243, 2006.

22. Rousseau JC and Delmas PD: Biological markers in osteoarthritis. Nat Clin Pract Rheumatol 3: 346-356, 2007.

23. Garnero P, Rousseau JC and Delmas PD: Molecular basis and clinical use of biochemical markers of bone, cartilage, and synovium in joint diseases. Arthritis Rheum 43: 953-968, 2000.

24. Clark KL, Sebastianelli W, Flechsenhar KR, Aukermann DF, Meza F, Millard RL, Deitch JR, Sherbondy PS and Albert A: 24-Week study on the use of collagen hydrolysate as a dietary supplement in athletes with activity-related joint pain. Curr Med Res Opin 24: 1485-1496, 2008.

25. Nagaoka I, Suzuki A, Kurokawa M, Tomonaga A, Fukagawa M, Watanabe K and Yamamoto T: Effect of a dietary supplement contatining collagen peptide on symptoms and biomarkers in individuals with knee pain. Gurukosamin Kenkyu (Glucosamine Res) 9: 40-47, 2013 (In Japanese).

26. Wu J, Fujioka M, Sugimoto K, Mu G and Ishimi Y: Assessment of effectiveness of oral administration of collagen peptide on bone metabolism in growing and mature rats. J Bone Miner Metab 22: 547-553, 2004.

27. Aito-Inoue M, Ohtsuki K, Nakamura Y, Park EY, Iwai K, Morimatsu F and Sato K: Improvement in isolation and identification of food-derived peptides in human plasma based on precolumn derivatization of peptides with phenyl isothiocyanate. J Agric Food Chem 54: 5261-5266, 2006.

28. Uehara K, Matsuo T, Nakashima M, Watanabe M, Nomura Y and Sugawara S: Effect of collagen hydrolystes on the progression of knee osteoarthritis. Gurukosamin Kenkyu (Glucosamine Res) 9: 53-60, 2013 (In Japanese).

29. Li P, Raitcheva D, Hawes M, Moran N, Yu X, Wang F, Matthews GL and Hawes M: Hylan G-F 20 maintains cartilage integrity and decreases osteophyte formation in osteoarthritis through both anabolic and anti-catabolic mechanisms. Osteoarthritis Cartilage 20: 1336-1346, 2012. 